\title{
In search of a primary outcome for community-based newborn infection trials in Eastern Uganda: a nested cohort study within the BabyGel pilot trial
}

\author{
N. A. Mobbs ${ }^{1,2^{*}}$, J. Ditai ${ }^{1,3}$, J. Abeso ${ }^{3,4}$, E. B. Faragher ${ }^{5}$, E. D. Carrol ${ }^{6}$, M. Gladstone $^{7}$, A. Medina-Lara ${ }^{8}$, \\ P. Olupot-Olupot ${ }^{9}$ and A. D. Weeks ${ }^{1}$
}

\begin{abstract}
Background: Due to their immature immune system, neonates are at high risk of infection. This vulnerability when combined with limited resources and health education in developing countries can lead to sepsis, resulting in high global neonatal mortality rates. Many of these deaths are preventable. The BabyGel pilot trial tested the feasibility of conducting the main randomised trial, with the provision of alcohol handgel to postpartum mothers for prevention of neonatal infective morbidity in the rural community. This secondary analysis sought to evaluate the methods of detecting infections in babies up to 3 months of age.

Methods: The pilot two-arm cluster randomised controlled trial took place in 10 villages around Mbale, Eastern Uganda. Women were eligible and recruited antenatally if their gestation was $\geq 34$ weeks. All infants of mothers participating in the BabyGel pilot trial were followed up for the first 3 months of life. Evidence for infant infection was collected using five different methods: clinician diagnosed infection, microbiologically confirmed infection, maternally reported infection, a positive infection screen using the World Health Organization (WHO) Integrated Management of Childhood Illness (IMCI) screening criteria, and reported antibiotic use identified during home and clinic visits. These methods were assessed quantitatively regarding the detection rates of suspected infections and qualitatively by exploring the challenges collecting data in the rural community setting.
\end{abstract}

(Continued on next page)

\footnotetext{
* Correspondence: mobbsnatalie@hotmail.co.uk

'Sanyu Research Unit, Department of Women's and Children's Health,

University of Liverpool and Liverpool Women's NHS Foundation Trust,

members of Liverpool Health Partners, Crown Street, Liverpool L8 7SS, UK

${ }^{2}$ University of Liverpool, Cedar House, Ashton Street, Liverpool L3 5PS, UK

Full list of author information is available at the end of the article
}

(c) The Author(s). 2019 Open Access This article is distributed under the terms of the Creative Commons Attribution 4.0 International License (http://creativecommons.org/licenses/by/4.0/), which permits unrestricted use, distribution, and reproduction in any medium, provided you give appropriate credit to the original author(s) and the source, provide a link to the Creative Commons license, and indicate if changes were made. The Creative Commons Public Domain Dedication waiver (http://creativecommons.org/publicdomain/zero/1.0/) applies to the data made available in this article, unless otherwise stated. 


\begin{abstract}
(Continued from previous page)
Results: A total of 103 eligible women participated in the BabyGel pilot trial, with 1 woman delivering twins. Of the 99 mother-infant pairs who consented to participate in the study, 55 infants were identified with infection in total. Maternal report of illness provided the highest estimate, with mothers reporting suspected illness for 45 infants $(81.8 \%$ of the total suspected infections identified). The WHO IMCI screening criteria identified 30 infants with suspected infection (54.5\%), and evidence for antibiotic use was established in 22 infants (40\%). Finally, clinician-diagnosed infection identified 19 cases (34.5\%), which were also microbiologically confirmed in 5 cases (9.1\%). Data collection in the rural setting was hindered by poor communication between mothers and the research team, limited staff awareness of the study in health centres resulting in reduced safeguarding of clinical notes, and widespread use of antibiotics prior to notification and clinical review. Furthermore, identification of suspected infection may not have been limited to severe infections, with ambiguity and no official clinical diagnosis being given to those identified solely by maternal report of infection.

Conclusions: A high rate of suspected infection was identified spanning the five sources of data collection, but no ideal method was found for detection of community neonatal infection. Although maternal self-reports of infant infection provided the highest detection rate, data collection via each source was limited and may have identified minor rather than major infections. Future studies could utilise the IMCI screening tool to detect severe community infection leading to referral for clinical confirmation. This should be combined with weekly contact with mothers to detect maternally suspected illness. Obtaining more details of the symptoms and timescale will improve the accuracy when detecting the total burden of suspected disease, and advising participants to retain medication packaging and prescriptions will improve identification of antibiotic use.
\end{abstract}

Trial registration: Babygel pilot trial - trial registration: ISCRCTN 67852437. Registered 02/03/2015.

Keywords: Paediatrics, Neonate, Infection, Uganda, Primary outcome, Diagnosis, Hand hygiene, Sepsis, Antibiotics

\section{Introduction}

Neonatal sepsis remains a global problem, causing $15 \%$ of all neonatal deaths [1] and claiming over 1.5 million infant lives annually [2]. These statistics may even be higher as neonatal mortality in developing countries is potentially under-reported by $>20 \%$ [3]. During the first year of life, an underdeveloped immune system renders infants particularly vulnerable to infection $[1,4,5]$. This vulnerability is further exacerbated in developing countries, such as Uganda, by limited resources and awareness. Despite the global burden of neonatal deaths declining, the African region still holds the highest neonatal mortality rate (NMR), with 30.5 per 1000 live births [6]. Since the initiation of the Millennium Developmental Goals, Sub-Saharan Africa has witnessed the largest relative decrease in under 5 mortality, yet still carries the burden of half the world's under 5 deaths and the proportion of those deaths within the first year of life increased [7]. The statistics highlight a demographic which drastically demands attention. High birth rates combined with high-risk newborn care practices and unhygienic environments including open rubbish dumps, roaming animals, and standing water may contribute to the high levels of morbidity and mortality. These settings with proportionately high and arguably preventable neonatal fatalities are those where affordable, acceptable, and sustainable interventions could yield the greatest and most immediate gains [1].
Low household wealth, lack of maternal education, and birth in rural settings are considered three critical determinants of NMR. A recent study in Eastern Uganda found the NMR to be higher in rural districts: 34 per 1000 compared with the national NMR of 20 per $1000(2013)[8,9]$. In Uganda, $42 \%$ of rural births are unattended [10], and combined with lack of sanitary equipment, limited access to clean water, potential lack of education, and unhygienic practices, risk of infection is high $[11,12]$. As many rural communities lack disposable amounts of clean water for hand washing, alcohol hand rub may offer the potential to reduce the rates of neonatal sepsis and improve the status of Africa's NMR [13].

The BabyGel study [ISRCTN67852437] (reported elsewhere) targeted a reduction in intrapartum and postnatal infection transmission by distributing alcohol hand rub to pregnant women antenatally in rural communities of Uganda for use during the birth and for 3 months postnatally [14]. The intervention aimed to prevent early-onset sepsis due to intrapartum maternal transmission and late-onset sepsis via human transmission of environmental pathogens.

Studies documenting rural community-acquired infection in neonates in Sub-Saharan Africa are scarce, largely due to the difficulties in data collection. No major studies have been done to identify rates of community-acquired infection including sepsis in rural Uganda $[15,16]$, despite $88 \%$ of the population residing in rural areas [10]. The 
only related studies conducted in Uganda's Mbarara Regional Referral Hospital and Mulago Hospital aimed to identify pathogenic organisms cultured from hospitalised septic neonates [2, 12]. Both studies found Staphylococcus aureus and Escherichia coli were the most common causative pathogens identified. Overall, the range of positive cultures was limited; $65 \%$ and $63 \%$ of pathogens were unidentifiable in Mbarara and Mulago hospitals respectively [2]. These limited results are reflected in similar studies in developing countries globally [17-20]. The obstacle to identifying the range of harmful pathogenic organisms is rooted in the fact that investigation strategies in the developing world are limited to blood and cerebrospinal fluid (CSF) cultures. Polymerase chain reaction (PCR) methods may yield greater results, but are generally unavailable $[21,22]$.

Although microbiological confirmation of infection is the gold standard for identifying neonatal sepsis, this cannot always be achieved in developing communities and diagnosis is often based solely on clinical signs [23]. Based on this, the Young Infants Clinical Signs Study Group (YICSSG) identified seven clinical signs which could be used with high sensitivity and specificity to predict requirement for neonatal referral, and WHO integrated this diagnostic screening tool in their guideline for the Integrated Management of Childhood Illnesses $[24,25]$. The validated IMCI screening form and diagnostic signs have since been adapted, utilised, and tested in many developing countries globally in rural settings to identify suspected infection [26, 27]. This screening tool was utilised in the BabyGel study to assess infectious morbidity.

This paper reports a secondary analysis of the pilot trial, comparing the various methods for identifying suspected infection in the initial 3 months of life in rural Ugandan communities.

\section{Methods}

\section{Study setting and participant recruitment}

The BabyGel pilot trial was conducted in 10 villages around Mbale, Uganda. Consent was sought from all mothers who were estimated to be at $>34$ weeks' gestation at the time of recruitment and resided in a participating village. A 3-month recruitment period was considered adequate by the investigators to assess the ability to recruit women to the study and resulted in recruitment of 103 women (who went on to deliver 104 newborns).

\section{Study timeline}

A timescale of 3 months was chosen to follow up the newborns/infants. This was chosen as a balance between the duration of time over which the provision of alcohol hand rub could be sustained and the period during which neonatal infection rates are highest and infant immunity is lowest. Vulnerability to infection is greatest during that early period, with one third of all child deaths occurring during the initial month of life [28].

\section{Data collection}

The gamut of suspected infection was identified using five methods of data collection: community-based IMCI screening forms, clinician-diagnosed infection detailed in hospital notes, microbiologically confirmed infection, and maternally reported infection and antibiotic use. The data collected was utilised to calculate the burden of disease amongst the participating infants in both the control and intervention groups.

Screening for suspected infection was carried out by the research team at the place of birth, in the village health worker (VHW) or parent's home, hospital, or health centre on every participating infant at day 1 and day 90 following delivery, as well as any infant with suspected illness in the interim (day 2-89). A day 1 screening form or interim visit/day 90 screening form which incorporated the WHO Integrated Management of Childhood Illness (IMCI) criteria for possible infection was utilised (Fig. 1) [29]. The use of a validated screening form standardised the information gathered, to identify what constituted an infected child and thereby prevent bias due to researchers subjectively diagnosing infection. The VHWs were trained for 1 day in the protocol and use of the screening form at the start of the study, and training was re-capped each time they returned a completed screening form to the research assistants. The IMCI screening form was utilised as the gold standard for infection data collection, allowing comparison with the other methods of detection.

A combination of passive and active surveillance methods was used to obtain the greatest amount of data possible. Participating women could contact the research team or an allocated VHW following delivery or if they suspected illness in their baby at any time postnatally, whilst the VHW and research team actively contacted the women around the expected due date for delivery, and 90 days from the day of birth. VHWs were encouraged to visit the mothers and complete the screening form twice weekly in the first month, and then weekly thereafter, although this was often not done. Midwives at the local health centres also notified the research team of inpatient births.

Screening data was collected electronically on handheld Samsung Galaxy S4 mobile smart phones using an electronic data capture system-the 'Open Datacollect Kit' (ODK). Researchers scanned the participants' individual barcode or entered their ID manually into the appropriate electronic form, prior to conducting the interview. On completion, the form was encrypted and 


\begin{tabular}{|c|c|c|c|}
\hline \multicolumn{4}{|l|}{ ASK: } \\
\hline Is the baby having difficulty with feeding? & Yes & No & \\
\hline Has the baby had convulsions (fits)? & Yes & No & \\
\hline \multicolumn{4}{|l|}{ LOOK, LISTEN, FEEL: } \\
\hline $\begin{array}{l}\text { Does the baby move only when stimulated (or have no movement at all)? } \\
\text { [Look at the baby's movements: } \\
\text { If the baby is sleeping, ask the mother to wake him/her. Does the baby move on its own? } \\
\text { If the baby is not moving, gently stimulate him / her. Does the baby not move at all?] }\end{array}$ & Yes & No & $\begin{array}{l}\text { If 'Yes' to } \\
\text { any of these } \\
\text { questions, }\end{array}$ \\
\hline Does the baby have severe chest wall indrawing? & Yes & No & the nearest \\
\hline $\begin{array}{l}\text { Does the baby have fast breathing (over } 60 \text { breaths per minute) } \\
\text { [Count the breaths in one minute. Repeat the count if more than } 60 \text { breaths per minute] }\end{array}$ & Yes & No & $\begin{array}{l}\text { health facility } \\
\text { for full } \\
\text { assessment }\end{array}$ \\
\hline $\begin{array}{l}\text { Is the baby's temperature over } 37.5^{\circ} \mathrm{C} \text { ? } \\
\text { [Take the temperature from under the baby's arm] }\end{array}$ & Yes & No & \\
\hline $\begin{array}{l}\text { Is the baby's temperature under } 35.5^{\circ} \mathrm{C} ? \\
\text { [Take the temperature from under the baby's arm] }\end{array}$ & Yes & No & \\
\hline
\end{tabular}

Fig. 1 The $\mathrm{IMCl}$ screening criteria for infection

transferred (via a server in Mbale) in real time to a data analyst at the University of Liverpool.

Any positive response to a question was considered an indicator of infection in the infant, and they were appropriately referred to the local health centre or Mbale Regional Referral Hospital (MRRH) where a clinical diagnosis, samples for culture, and treatment could be obtained. Where possible, clinical notes delineating the physician diagnosis and any associated microbiological evidence were then collected for all infants admitted.

Where expertise and facilities allowed, blood samples were collected, alongside stool and pus samples where relevant. Samples were analysed at the microbiology laboratory of Busitema University. Blood was aseptically collected into BACTEC bottles using the vacutainer system by trained laboratory personnel and loaded with inoculum into the BACTEC 50 blood culture system for auto-detection of microbial growth. Those with positive growth were sub-cultured on MacConkey agar (Oxoi$\mathrm{d}$-UK), blood agar, and chocolate agar (Oxoid-UK) for maximum recovery of bacteria. A portion of the stool samples were inoculated in selenite $\mathrm{F}$ broth, incubated at $37^{\circ} \mathrm{C}$ for $18 \mathrm{~h}$, and then sub-cultured on XLD agar and MacConkey agar. The other portions were directly inoculated on XLD agar and MacConkey agar. Pus swabs were inoculated on blood agar, MacConkey agar, and chocolate agar. All the inoculated plates were incubated at $37^{\circ} \mathrm{C}$ for $18-24 \mathrm{~h}$ under ambient air except for chocolate agar plates which were incubated at $37{ }^{\circ} \mathrm{C}$ in an anaerobic jar. Isolated bacteria were identified using standard biochemical methods and sensitivity tested against a range of locally available antibiotics. Samples were not obtained from infants with antibiotic exposure prior to health centre admission as there is a low detection rate with such samples.
Data was collected on the use of antibiotics. The BabyGel pilot trial interim screening form specifically asked respondents to name any medicines that had been used, and VHWs and/or other health workers were also asked to comment on antibiotic use in free text on the screening tool. Clinical notes were also reviewed to collect written data on drugs administered to the infant during and prior to admission. When mothers retrospectively commented on antibiotic use, they were asked if they had kept the box or prescription for confirmation, or if they could recall the drug administered to the child; cases of uncertainty were not counted, e.g. 'baby was taken to a nearby drug shop and dispensed unknown syrup'.

The same screening form was used at day 90 and during the interim period; it questioned: 'Has your baby been unwell since the last visit?', Has your baby ever been admitted to hospital since the last visit?', 'How many times?' (for each), and 'For how long did your baby spend in the hospital?'. These questions were designed to capture any infants who had been ill but had screened negative or in whom clinical notes were not collected. Further details to elaborate were also recorded in a free-text box completed by the interviewer at the end of the form.

Finally, an international standard verbal autopsy questionnaire was utilised to determine the cause of death in any infant that died during the study.

\section{Ethical considerations}

This secondary analysis was part of the BabyGel pilot trial, granted ethical approval by the 'University of Liverpool Research and Ethics Committee' in the UK, the 'MRRH Institutional Review Committee' in Mbale, and the Uganda National Council for Science and Technology 
(RETH000808, REIRC IN - COM 011/2015 and HS1768 respectively).

Participant consent interviews were conducted in the language most comprehensible to the subject (local language Lumasaba or English), and written consent was subsequently obtained.

\section{Results}

A total of 103 mothers consented to participate in the study (104 infants-including one set of twins). Three stillbirths, an early neonatal death with no screening, and a mother who withdrew from the study with no screening were all excluded; one late neonatal death with completed day 1 screening details and a mother-infant pair who relocated after completing the day 1 screening were retained in the statistical analysis. Thus, a total of 98 women (99 mother-infant pairs) were included, with 96 women (97 mother-infant pairs) completing 3 months of follow-up (Table 1). The mean age of the women who participated in the study was 24.9 years (mean (s.d.) 5.7 [15-38]).

The study identified evidence for suspected infection in $55(55.6 \%)$ of the 99 infants followed up, of which 27

Table 1 Demographics of study participants

\begin{tabular}{ll}
\hline & $N(\%)$ \\
\hline Total participants & 103 \\
$15-20$ & \\
$21-30$ & $25(24.3)$ \\
$31-40$ & $56(54.4)$ \\
Mean age & $21(20.4)$ \\
Marital status & 24.9 \\
Single & \\
Married & $22(21.4)$ \\
Divorced/separated & $80(77.7)$ \\
Widowed & 0 \\
Highest level of education & $1(1.0)$ \\
No formal education & \\
Did not complete primary education & $3(2.9)$ \\
Completed primary (PLE) & $52(50.5)$ \\
Completed ordinary or advanced level (UCE/UACE) & $32(31.1)$ \\
Completed diploma or degree & $14(13.6)$ \\
Primary occupation & $2(1.9)$ \\
Peasant farmer, no paid employment, or housewife & $95(92.2)$ \\
Student & $3(2.9)$ \\
Business woman & $2(1.9)$ \\
Professional & $1(1.0)$ \\
\hline & $2(1.9)$ \\
\hline
\end{tabular}

*Data missing on 1 woman and 28 infants were in the control and interventions arms respectively. The clinical comparative outcomes are reported elsewhere. Figure 2 summarises the results of the study. Considering the 99 infants followed up, in total, 45 infected infants (45.5\%) were identified by maternal report, 30 (30.3\%) screened positive, and clinical notes were obtained for 19 (19.2\%) (of which 5 cases (5.1\%) were supported by microbiological evidence). In some cases, infection was positively identified from multiple sources; however, evidence from all three of these sources was only obtained for $12 / 55(21.8 \%)$ of the total infants identified with suspected infection (Fig. 3). There were no infection outbreaks in the region at the time of the study.

Of the 55 infants with evidence of suspected infection, IMCI screening forms were positive for indicators of infection for 30 infants $(54.5 \%)$; $22(40 \%)$ infants had a single positive screen and $8(14.5 \%)$ had two positive screens during the follow-up period (mean (s.d.) number of positive screens per infant: $1.27(0.45)$ ). There were 8 positive screens at the day 1 follow up, 3 at the day 90 follow up, and 27 screened positive for suspected infection during the interim period (Table 2). Reliable dates were obtained for only 11 of the 27 positive screens during the interim period; of these, $2(18.2 \%)$ occurred in the first 14 days after birth, 4 (36.4\%) occurred between day 15 and day 30, and the remaining 5 (45.5\%) during months 2 and 3 of the follow-up period (mean (s.d.) age of infants at positive screen: 40 (32) days). A relapse was considered any second positive screen $<7$ days after the first positive screen, and a new episode of infection was any second positive screen $>7$ days after the first. Of the 8 babies with two positive screens during the study, 3 screened positive at day 1 and during the interim ( 2 unreliable dates and 1 relapse), 2 screened at day 90 and during the interim ( 1 unreliable date and 1 episode of new infection), and 3 screened positive twice during the interim period ( 2 unreliable dates and 1 relapse).

Table 3 illustrates the various fields which indicated infection in infants on each screening form. The remaining 25 infected infants were never captured on any screening form, but were identified via clinical notes and maternal report. In this way, successful use of the IMCI screening form criteria was limited; some of these infants screened negative yet were subsequently referred to a health centre and clinically diagnosed with suspected infection.

Medical notes were collected for 19 infants who were clinically diagnosed with infection (Table 2), of which 12 had also screened positive for indicators of infection on a screening form (Fig. 3). The VHWs and study researchers could not attend for referral with all infants who screened positive, limiting the amount of medical notes that could be traced. One neonate died within the 


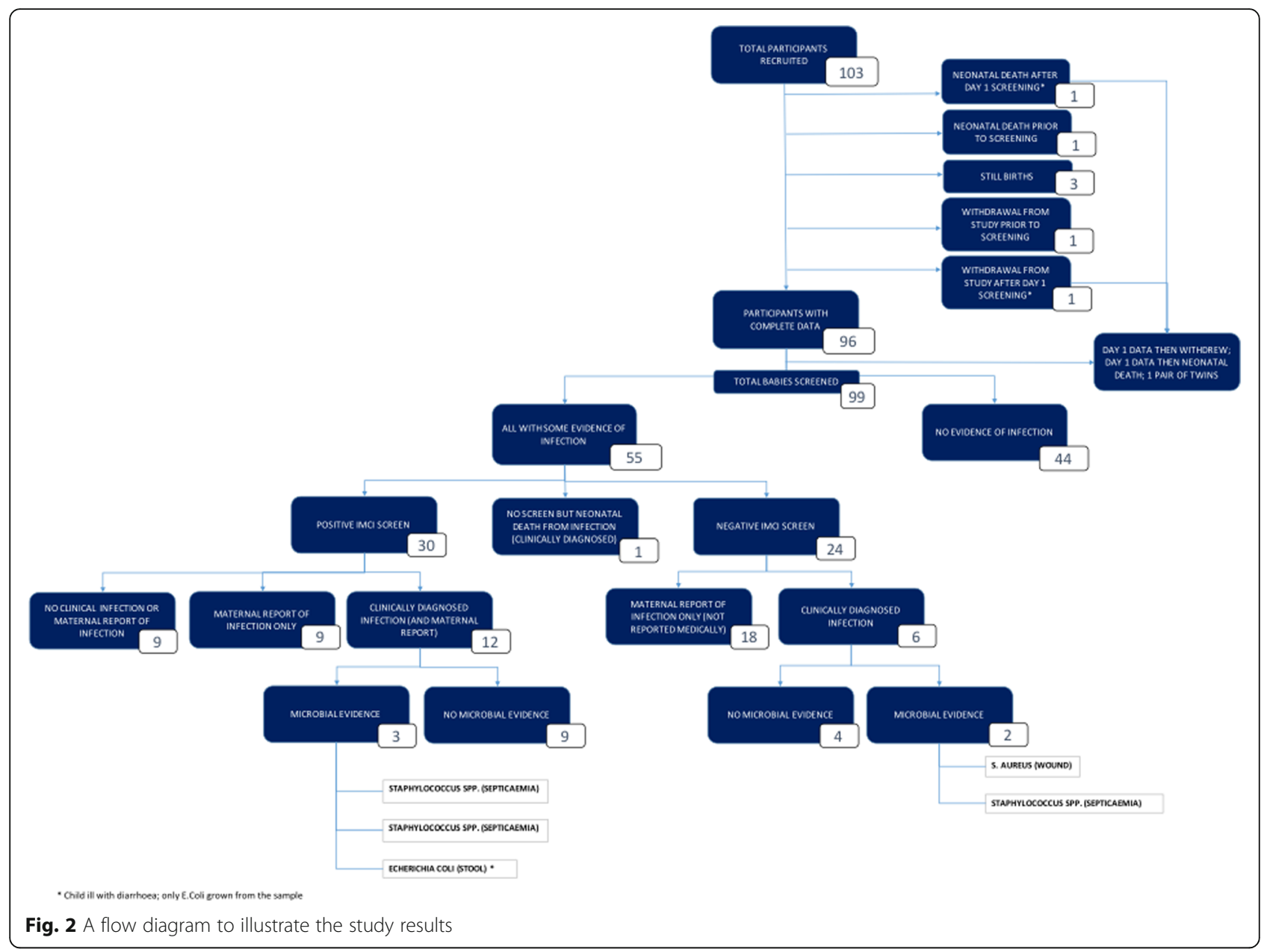

first month. The suspected cause of death was 'sepsis' based on the information gathered in the clinical notes and verbal autopsy form.

It was not possible to obtain lab samples for all 19 infants due to limited resources and staff at the health centres. In total, 16 culture samples were obtained from 10 of these infants, of which 5 samples returned positive results (Table 4). The most common pathogen cultured was Staphylococcus spp. (60\%), and both Escherichia coli and Staphylococcus aureus were cultured once each (20\% respectively). As anticipated, positive cultures were only obtained in $31 \%$ of samples: 3 blood samples, 1 stool sample, and 1 pus sample. No CSF samples were drawn (due to limited resources at the point of care). In cases whereby multiple samples were taken from infants (e.g. blood and pus), growth only occurred in 1 sample.

A total of 45 mothers reported that their child had been infected on the 90-day IMCI screening form or interim form. Of these, 21 infants also screened positive on a screening form; therefore, the question captured 24 cases whereby infants may have been ill but were not additionally screened when ill/did not screen positive on a screening form (Fig. 3). Mothers reported 106 counts of illness in total, due to some infants falling ill on multiple occasions. The IMCI screening forms therefore failed to identify 69 occasions in which infants may have been infected. Data collected via maternal reports of infection identified $45 / 55$ (81.8\%) of the total infants with suspected illness.

There is evidence for 22 infants being administered antibiotics, in some cases prior to hospital admission. Table 5 indicates which infants were subject to antibiotic use and where this information was gathered from, and it also lists the types of antibiotics in use and in what frequency used. In total, 8 infants were also clinically diagnosed with malaria (Table 2). All were in the post-neonatal period and received antimalarial medication (14.5\% of the total with suspected infection); 3 cases were confirmed with a rapid diagnostic screening test, but there was no evidence of diagnostic testing for the other 5 infants. Co-infection with pneumonia occurred in 1 infant, and a skin infection in another.

A total of 15 mothers were known to carry HIV, of which 4 were on co-trimoxazole prophylaxis. None of 


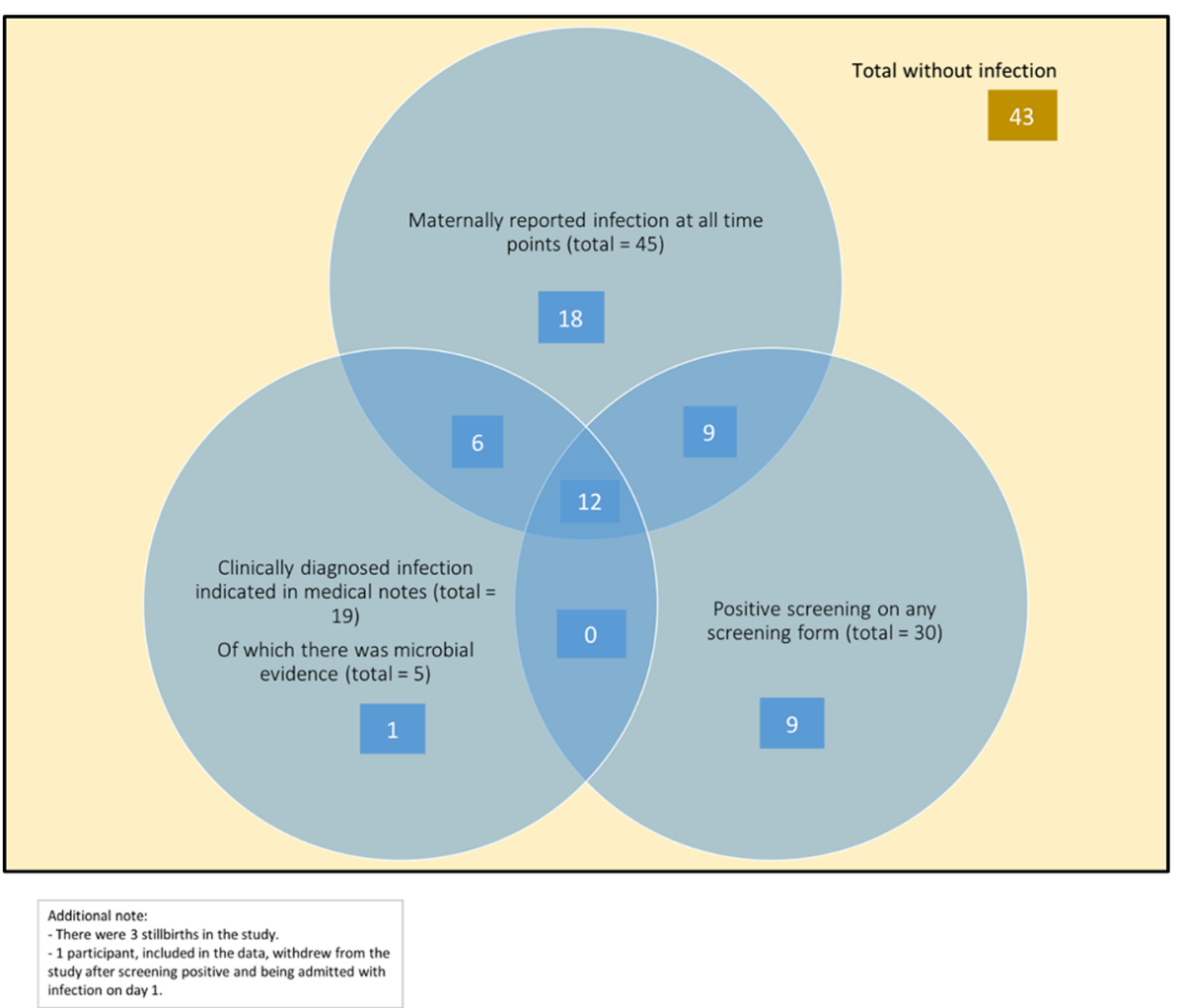

Fig. 3 Venn diagram indicating the various sources by which presumed infection was identified in participants

the babies of HIV-infected women received prophylaxis. The infection rate in babies of HIV-infected mothers was $73 \%$ compared to $57 \%$ in those of non-infected mothers ( $p=0.236$, chi-squared test).

In order to calculate the sensitivity and specificity, clinical diagnosis was utilised as the gold standard (despite there being $80.8 \%$ of missing results), assuming that all missing results were negative. With these provisos, the results were:

Maternally reported infection: sensitivity $18 / 19$ (94.7\%: $74.0 \%$ to $99.9 \%$ ), specificity $53 / 80$ (66.3\%: $54.8 \%$ to 76.4\%); positive IMCI screen: sensitivity $12 / 19$ (63.2\%: $38.6 \%$ to $83.7 \%)$, specificity $62 / 80$ (77.5\%: $66.8 \%$ to 86.1\%); evidence of antibiotic use: sensitivity $17 / 19$ (89.5\%: $66.9 \%$ to $98.7 \%$ ), specificity $75 / 80$ (93.8\%: $86.0 \%$ to $97.9 \%)$.

\section{Discussion}

\section{Summary of results}

Utilising all five methods of data collection, suspected infection was identified in $55.6 \%$ of the study participants, a high figure especially when considering some of these infants were ill on multiple occasions. None of the methods of data collection successfully identified all cases of suspected neonatal infection. The IMCI screening forms successfully identified infection in $30.3 \%$ of the total study participants and $54.5 \%$ of the total number of infants with suspected infection. Evidence for infection from clinical notes was found for only $34.5 \%$ of the infants with suspected infection, and microbiological evidence was obtained for only $9.1 \%$ of the total suspected with infection. Similar to reports in previous studies, Staphylococcus spp. and Escherichia coli were the most common causative pathogens identified $[2,12]$. Maternal report of infection captured the greatest amount of data, as mothers reported illness for $81.8 \%$ of the total presumed infected infants. Finally, evidence for antibiotic use was only found in $40 \%$ of the total infected infants.

Whilst it is desirable to have the sensitivity and specificity for each method of data collection, this had to be calculated (and must also be interpreted) with great caution. The analysis was calculated on all 99 infants (including those who did not test positive) and assumes, probably erroneously, that the absence of a positive result for any test method equated to a negative test result. Furthermore, the evidence available in the clinical notes had to be utilised as the gold standard and that itself is suspect.

\section{Comparison to other studies}

The main difference between this study and others was the prospective rather than retrospective element. A 
Table 2 Results of the study for all participants

\begin{tabular}{|c|c|c|}
\hline & $N$ & $\%(95 \% \mathrm{Cl})$ \\
\hline Total maternal recruits & 103 & \\
\hline Withdrawals & 2 & \\
\hline Stillbirths & 3 & \\
\hline Neonatal deaths & 2 & \\
\hline Total infants screened + followed up* & 99 & \\
\hline Any evidence of infection & 55 & $55.6(45.2: 65.5)$ \\
\hline \multicolumn{3}{|l|}{ Positive screen } \\
\hline Day 1 & 8 & $8.1(3.6: 15.3)$ \\
\hline Interim & 27 & $27.3(18.8: 37.1)$ \\
\hline Day 90 & 3 & $3.0(0.6: 8.6)$ \\
\hline Day 1 and/or interim and/or day 90 & 30 & $30.3(21.5: 40.4)$ \\
\hline Clinical diagnosis of infection (from clinical notes) & 19 & $19.2(12.0: 28.3)$ \\
\hline Oomphalitis & 1 & $1.0(<0.1: 5.5)$ \\
\hline Pneumonia & 2 & $2.0(0.2: 7.1)$ \\
\hline Diarrhoea & 1 & $1.0(<0.1: 5.5)$ \\
\hline Neonatal septicaemia & 6 & $6.1(2.3: 12.7)$ \\
\hline Upper respiratory tract infection & 3 & $3.0(0.6: 8.6)$ \\
\hline Skin pustules/infection & 3 & $3.0(0.6: 8.6)$ \\
\hline Evidence from all three sources & 12 & $12.1(6.4: 20.2)$ \\
\hline Unspecified source & 3 & $3.0(0.6: 8.6)$ \\
\hline Microbiological evidence & 5 & $5.1(1.7: 11.4)$ \\
\hline Maternally reported infection & 45 & $45.5(35.4: 55.8)$ \\
\hline Received antibiotics & 22 & $22.2(14.5: 31.7)$ \\
\hline Infants receiving antimalarial medication & 8 & $8.1(3.6: 15.3)$ \\
\hline
\end{tabular}

$\mathrm{Cl}$ confidence interval

*Includes 1 set of twins, 1 neonatal death (screened at day 1), and 1 withdrawal (screened at day 1)

recent cross-sectional study in Eastern Uganda involved conducting face to face interviews with 2237 women who had delivered in the past 12 months, to determine the neonatal mortality rate and influential factors [30]. The retrospective nature of the study meant it was therefore subject to recall bias, and the sole reliance of maternal assessment of 'danger signs at birth' (e.g. 'yellow skin', 'difficulty breathing') was arguably subjective and potentially unreliable. A similar community-based prospective cohort study was carried out in Mbale, Eastern Uganda, to determine perinatal mortality and risk
Table 4 Outcome for culture samples obtained

\begin{tabular}{lll}
\hline & Organisms & $N(\%)$ \\
\hline Total samples taken & & 16 \\
Blood samples & & $10(62.5)$ \\
Pus swab & & $2(12.5)$ \\
Stool sample & $4(25.0)$ \\
Cerebrospinal fluid & & 0 \\
Total positive samples & & $5(31.3)$ \\
Positive blood cultures & Staphylococcus spp. (3) & $3(18.8)$ \\
Positive pus swabs & Staphylococcus aureus (1) & $1(6.3)$ \\
Positive stool samples & Escherichia coli (1) & $1(6.3)$ \\
Cerebrospinal fluid & & 0 \\
\hline
\end{tabular}

factors of perinatal death for 7 days postnatally in 835 women [9]. There were 34 perinatal deaths (18 excluding stillbirths), none of which were officially registered or had death certificates (again illustrating the difficulty of monitoring infection and NMR using the accepted estimated statistics for the country). Again, the women were interviewed within 4 weeks of delivery to recall their antenatal care attendance, socio-demographic characteristics, and infant mortality. Other studies which considered community infection in neonates generally required the women to attend hospital or antenatal clinics for data collection, due to the difficulty of regularly monitoring infants in the community home.

\section{Limitations of study}

The prospective element of this study meant data had to be collected in real time, and the obstacles to data collection faced in this trial highlight why there is a paucity of published research on rural Ugandan mothers and children. Numerous challenges prevented widespread use of the screening forms. The VHWs were not always notified when infants fell ill and may not have been available to visit the mother at home. This likely resulted in cases whereby infection was missed. Mothers also often bypassed the study screening process, attending hospital or health centres directly with their children without notifying the research team, presumably due to concerns about their baby's health and to avoid delays in seeking care. Others screened positive on the IMCI form, but there was no record of their subsequent

Table 3 Criteria indicating presumed infection in infants (based on IMCl screening forms)

\begin{tabular}{|c|c|c|c|c|c|c|c|}
\hline Day & Difficulty feeding & Convulsions & $\begin{array}{l}\text { Moving only when } \\
\text { stimulated / not at all }\end{array}$ & $\begin{array}{l}\text { Chest wall } \\
\text { indrawing }\end{array}$ & $\begin{array}{l}\text { Fast respiratory } \\
\text { rate }\end{array}$ & $\begin{array}{l}\text { High axillary } \\
\text { temperature }\end{array}$ & $\begin{array}{l}\text { Low axillary } \\
\text { temperature }\end{array}$ \\
\hline 1 & 5 & 0 & 0 & 0 & 0 & 4 & 0 \\
\hline 90 & 1 & 1 & 1 & 1 & 0 & 2 & 0 \\
\hline Interim (day 2-89) & 9 & 3 & 2 & 5 & 19 & 11 & 1 \\
\hline Totals & 15 & 4 & 3 & 6 & 19 & 17 & 1 \\
\hline
\end{tabular}


Table 5 A table indicating antibiotic use in all infants with suspected infection

\begin{tabular}{|c|c|c|}
\hline & $N$ & $\%$ \\
\hline Total participants with infection & 55 & 100 \\
\hline Total antibiotic exposure & 22 & $40.0(27.0: 54.1)$ \\
\hline \multicolumn{3}{|l|}{ Sources of evidence for antibiotic use } \\
\hline Clinical notes & 17 & $30.9(19.1: 44.8)$ \\
\hline $\begin{array}{l}\text { Direct question addressing use of } \\
\text { any medication on } \mathrm{IMCl} \text { form }\end{array}$ & 3 & $5.5(1.1: 15.1)$ \\
\hline $\begin{array}{l}\text { Comments on antibiotic use (free } \\
\text { text) }\end{array}$ & 19 & $34.5(22.2: 48.6)$ \\
\hline Total antibiotic exposures & $52^{*}$ & 100 \\
\hline \multicolumn{3}{|l|}{ Named antibiotic } \\
\hline Amoxicillin & 8 & $15.4(6.9: 28.1)$ \\
\hline Ceftriaxone & 10 & $19.2(9.6: 32.5)$ \\
\hline Gentamycin & 7 & $13.5(5.6: 25.8)$ \\
\hline Metronidazole & 3 & $5.8(1.2: 15.9)$ \\
\hline Ampiclox & 4 & $7.7(2.1: 18.5)$ \\
\hline Nalidixic acid & 1 & $1.9(0.1: 10.3)$ \\
\hline Ampicillin & 4 & $7.7(2.1: 18.5)$ \\
\hline Cloxacillin & 2 & $3.8(0.5: 13.2)$ \\
\hline Benzyl penicillin & 2 & $3.8(0.5: 13.2)$ \\
\hline Co-trimoxazole & 6 & $11.5(4.4: 23.4)$ \\
\hline Neomycin & 1 & $1.9(0.1: 10.3)$ \\
\hline Amikacin & 1 & $1.9(0.1: 10.3)$ \\
\hline Mupirocin & 1 & $1.9(0.1: 10.3)$ \\
\hline Tetracycline & 1 & $1.9(0.1: 10.3)$ \\
\hline Erythromycin & 1 & $1.9(0.1: 10.3)$ \\
\hline
\end{tabular}

*Many babies had more than 1 antibiotic

attendance at the study health centres as should have happened per protocol. Mothers may have taken their infants to local pharmacies or private clinics to prevent the informal charges that are sometimes demanded by health centre staff. Furthermore, mothers were sometimes absent from home during day 1 or 90 screening, and others failed to notify researchers that their child had been born for some time. All these presented enormous difficulties in collecting accurate, reliable data on infant infection.

Although clinical notes were obtained where possible from hospitals and health centres following referral, delays in researcher notification meant that clinical notes were often no longer traceable. Finite members of staff at the health centres and hospitals were notified of the study, and therefore, the safeguarding of participant notes was limited by staff awareness. This was an important learning point from the pilot study and may have been exacerbated by parental refusal to attend hospital following referral which has been documented in previous community-based studies [20,31].
Unlike the IMCI screening forms, and clinical or microbiological data which made diagnoses in real time, maternal report of infection captured the greatest number of infants with suspected infection, but was subject to recall bias. Likewise, data obtained on antibiotic exposure was occasionally gathered from free-text comments on the screening forms and therefore also subject to recall bias.

Furthermore, the IMCI form is a validated tool for severe illness, whilst the other methods used in this study are more specific for infections. This makes comparison of infection rates challenging. Mothers were asked: 'Has your baby been unwell since the last visit?' 'How many times has baby been unwell?' and 'Has your baby ever been admitted to the hospital since the last visit?'. Besides recall bias, the closed questions neither establish the severity of the suspected infection nor the time period. Thus, it would be possible for the quoted rates to include both minor infections and relapses rather than individual episodes of severe illness. Only 18 of the 45 infants (40\%) identified via maternal report were clinically diagnosed. This can be partially explained by lack of care-seeking at health facilities and the difficulty in collecting clinical documentation from busy health centres and might also indicate limitations in maternal recognition and report of severe infection.

Collecting hard evidence for infant infection was challenging. Whilst clinical notes reported infections ranging from neonatal septicaemia to skin pustules and diarrhoea, microbiological evidence for infection was very limited. Although funding for tests was made available for the study, established local practice is not to rely on microbiological testing but rather to provide empirical treatment. Furthermore, in Mbale, antibiotics can be obtained with ease over the counter from local sources without a qualified diagnosis being made. Antibiotic use could therefore not be confidently utilised as an indicator of infection (and especially severe infection) in participants, even though the information was easily obtained retrospectively from mothers.

The free-text comments documented on the screening forms indicated liberal use of freely available antibiotics within the rural setting without a prescription. Antibiotic exposure prior to admission meant limited samples could be obtained from infants, resulting in few cases of illness with microbiological evidence. Although $31 \%$ of culture samples obtained were positive (comparable to the 35 and 37\% obtained in the Mbarara Regional Referral Hospital and Mulago Hospital studies respectively) $[2,12]$, only 10 infants with suspected illness had samples taken for culture. Furthermore, the process of transporting samples was not without error: one baby had samples taken for complete blood count (CBC) with blood culture and sensitivity which was delivered to the 
hospital where there were no personnel to receive it. The sample subsequently haemolysed and was rejected due to a public holiday resulting in the sample remaining at room temperature for $>48 \mathrm{~h}$.

A further reason for the limited number of samples was the lack of skilled staff. Hospitals and health centres in Uganda are often under-staffed, and therefore, necessary tests and investigations cannot be carried out as required, or advised by local protocol. Arguably, this is one of the greatest challenges of carrying out research of this type in this environment. With appropriate funding, it would be possible to set up a study in a high-quality European standard paediatric ward with all the associated resources, but this would ultimately alter the study setting and prevent this being a pragmatic study.

\section{Strengths of study}

The study had a high follow-up rate, with all participants being tracked to day 90 postnatally, except the women who withdrew their consent or relocated and those with perinatal deaths. The prospective format gives a variety of strengths: the potential for clear temporal chronology to link the outcome to the exposure, limited risk of recall bias due to rapid assessment with the IMCI screening forms, and clinical or microbiological diagnosis in real time. The variety of methods utilised for data collection meant that a spectrum of illness was captured. In cases whereby multiple methods successfully identified singular episodes of infection, the combined data was complementary, creating a sound clinical picture, which could be utilised to differentiate severe from minor infection.

\section{Future recommendations}

In future studies, alterations must be made to the methods of data collection and the details gathered. The choice of method will depend on a balance of cost, feasibility, and acceptability. Unless all methods measure severe infection only, differences in infection risk will be incomparable; limiting the study outcome to major infections would permit comparative analysis. The IMCI screening form should only identify severe infection, as should the clinical notes of those presenting directly to health care providers. In contrast, the maternal report of infection captured a huge number of infants with potentially minor or suspected infections. This was compounded by the difficulty of VHWs accessing the community homes making it difficult to verify maternal reports. In order to reduce recall bias and ensure the infections being identified are severe, the maternal questionnaire about recent infections should include greater detail on the symptoms, signs, date, and length of illness. Regular, weekly communication with participants via a toll-free number would improve communication between the researchers and mothers to determine if the child has been ill in the past week (whilst also reducing the risk of recall bias). A researcher could attend the home to assess the ill child or visit the health centre/hospital the mother attended during the week to collect clinical notes if the baby was admitted, to ensure as many infants with suspected infection are captured as possible. Particular attention should be paid around the expected date of delivery and final follow-up, with frequent communication to guarantee adequate data collection.

To improve the collection of both clinical and microbiological data, improved communication between the health centres and laboratory alongside more reliable services will be required for future studies. Although multiple samples were obtained from infected infants, only singular samples yielded results, confirming the importance of obtaining multiple samples where possible and relevant. This thorough method optimised the chance of achieving measurable pathogenic growth and should continue to be utilised in future studies. As many members of clinical staff as possible should be notified of the research taking place at the health centres and local hospital, so they have an awareness of the mothers participating and can notify researchers, keeping notes and samples relating to the participants safe. Where screening forms result in a referral to a health centre or hospital, researchers should attend with the infant in order to collect the clinically relevant notes and make the health practitioners aware that the infant is participating in the study.

The challenge of antibiotic use prior to hospital admission and self-prescribed antibiotic use within the community is difficult to address. During the 90-day interview, numerous mothers described occasions on which their child had been ill, and they had self-medicated them and/or not notified the VHWs. Future studies could monitor antibiotic use reported both verbally and with evidence of prescription or drug packaging to more accurately capture the cohort of infants with suspected infection. Administration of antibiotics is a useful indicator of how ill the mother deems their child to be, particularly as a limitation of maternal report of infection is the risk of identifying minor illness rather than severe infections. The benefit of capturing data through maternally reported antibiotic use includes identifying suspected infection regardless of where the care was sought and would be helpful to monitor as a public health outcome given the financial cost of antibiotic use and development of antibiotic resistance, which is a growing international concern [20,32]. Drawbacks include a potential overestimation of the frequency of true infections and difficulty in ascertaining which drugs were administered as often drugs are provided over the counter without a prescription. This 
might be addressed by ensuring prescriptions and packaging are retained for inspection by the research team. Alternatively, to reduce the risk of overestimation, evidence for antibiotic use could be restricted to that provided by qualified health care professionals. The downside would be that it might also result in cases being missed. There is currently a paucity of data on the use of antibiotics within Uganda's rural communities [33], and monitoring this in future would be valuable [34, 35].

\section{Conclusions}

Rural communities are often relatively inaccessible and isolated, and antibiotics are frequently obtained over the counter without a prescription. Formal clinical diagnoses and laboratory-based evidence are rare, due to antibiotic use prior to admission and limited medical and laboratory facilities. There were limitations to each method of infection ascertainment, yet overall, each method captured some evidence for suspected infection, in some cases creating a clinically complementary picture. It follows that each method of data collection utilised in this study could be beneficial if used in future studies with some considerations. Future studies should focus on the clinical diagnosis of severe infection. The IMCI screening tool is a validated method of picking up severe illness and should continue to be used as a means of detecting community infection and directing referral, with clinical diagnosis being used for confirmation. Screening should be improved with weekly contact with the mothers to detect maternally suspected illness. Greater detail of the symptoms and timescales would enhance the accuracy of detection and enable maternal reports of infection to more confidently indicate the total burden of suspected disease of relevant severity. Finally, communication between researchers, health care staff, and participants is crucial, so as to provide high-quality data on infective morbidity.

\section{Abbreviations \\ CBC: Complete blood count; CSF: Cerebrospinal fluid; IMCI: Integrated Management of Childhood Illnesses; MRRH: Mbale Regional Referral Hospital; NMR: Neonatal mortality rate; ODK: Open Datacollect Kit; PCR: Polymerase chain reaction; VHW: Village health worker; WHO: World Health Organization; YICSSG: Young Infant Clinical Signs Study Group}

\section{Acknowledgements}

We would like to thank Nathan M. Odeke who assisted with data collection early in the process; and Andrew Bennett who assisted with the initial data analysis.

\section{Funding}

Funding was received from the Medical Research Council/Wellcome Trust/ DfID via the Global Health Trials Scheme (Reference: MR/MO17990/1). The funding body did not participate in the study design, collection, analysis, or interpretation of data, nor contribute to writing the manuscript in any way.

\section{Availability of data and materials}

The current paper is a secondary analysis nested within the Babygel pilot trial. The datasets generated and analysed during the Babygel pilot trial are not publicly available at present but will be made available on request from the Sanyu Research Unit, Department of Women's and Children's Health, University of Liverpool. These requests should be directed to Professor A. Weeks (aweeks@liverpool.ac.uk).

\section{Authors' contributions}

ADW conceived the idea for the paper as part of a secondary analysis of the BabyGel pilot study. Data appropriate to the nested study was refined by JD, ADW, BEF, and NAM from the BabyGel pilot trial datasets. NAM created all tables and Figs. 2 and 3 with critical review from ADW. NAM developed the first draft which was initially reviewed by ADW. NAM, JD, JA, EDC, MG, AML, and POO were involved with critical review of subsequent drafts. All authors read and approved the final manuscript.

\section{Ethics approval and consent to participate}

This study was nested within the Babygel pilot trial; performed in accordance with the Declaration of Helsinki and granted ethical approval by the University of Liverpool's Research and Ethics Committee, Mbale Regional Referral Hospital Institutional Review Committee and the Uganda National Council for Science and Technology (RETH000808, REIRC IN - COM 011/2015 and HS1768 respectively). Informed consent to participate was obtained from all participants prior to recruitment. The study was registered with the ISRCTN registry (ISCRCTN 67852437; 18/03/2015).

\section{Consent for publication}

Not applicable.

\section{Competing interests}

The authors declare that they have no competing interests.

\section{Publisher's Note}

Springer Nature remains neutral with regard to jurisdictional claims in published maps and institutional affiliations.

\section{Author details}

'Sanyu Research Unit, Department of Women's and Children's Health, University of Liverpool and Liverpool Women's NHS Foundation Trust, members of Liverpool Health Partners, Crown Street, Liverpool L8 7SS, UK. 2University of Liverpool, Cedar House, Ashton Street, Liverpool L3 5PS, UK. ${ }^{3}$ Sanyu Africa Research Institute (SAfRI), Mbale Regional Referral Hospital, Pallisa-Kumi Road Junction, P.o Box 2190, Mbale, Uganda. ${ }^{4}$ Department of Paediatrics, Mbale Regional Referral Hospital, Mbale, Uganda. ${ }^{5}$ Tropical Clinical Trials Unit, Liverpool School of Tropical Medicine, Pembroke Place, Liverpool L3 5QA, UK. ${ }^{6}$ Department of Clinical Infection, Microbiology and Immunology, Institute of Infection and Global Health, University of Liverpool, 8 West Derby Street, Liverpool L69 7BE, UK. ${ }^{7}$ Department of Women's and Children's Health, Institute of Translational Medicine, University of Liverpool and Alder Hey NHS Foundation Trust, members of Liverpool Health Partners, Eaton Road, Liverpool L12 2AP, UK. ${ }^{8}$ Health Economics Group, University of Exeter, Exeter, UK. ${ }^{9}$ Faculty of Health Sciences, Busitema University, P.o Box 1460, Mbale, Uganda.

Received: 20 March 2018 Accepted: 25 February 2019

Published online: 13 March 2019

References

1. United Nations Children's Fund (UNICEF): Division of Data, Research and Policy. Committing to Child survival: A promise renewed. Progress Report 2014. https://data.unicef.org/wp-content/uploads/2015/12/APR-2014170ct14-web_194.pdf. Accessed 3 Apr 2017.

2. Kiwanuka J, Bazira J, Mwanga J, Tumusiime D, Nyesigire E, Lwanga N, et al. The microbial spectrum of neonatal sepsis in Uganda: recovery of cultural bacteria in mother-infant pairs. PLoS One. 2013;8(8):e72775. https://doi.org/ 10.1371/journal.pone.0072775.

3. Vergnano S, Sharland M, Kazembe P, Mwansambo C, Heath PT. Neonatal sepsis: an international perspective. Arch Dis Child Fetal Neonatal Ed. 2005; 90:F220-4. https://doi.org/10.1136/adc.2002.022863.

4. Cant AJ, Gennery AR. Neonatal infection: immunodeficiency. In: Rennie JM, editor. Robertson's textbook of neonatology. 4th ed. Philadelphia: Elsevier Churchill Livingstone; 2005. p. 993-1011. 
5. Levene MI, Tudehope DI, Thearle J. Essentials of neonatal medicine. 3rd ed. Oxford: Blackwell Science; 2000.

6. World Health Organisation: WHO Department of Health Statistics and Information Systems of the Health Systems and Innovation Cluster. World Health Statistics. Statistics Report 2015. http://apps.who.int/iris/bitstream/ 10665/170250/1/9789240694439_eng.pdf?ua=1\&ua=1. Accessed 3 Apr 2017.

7. United Nations. In: Way C, editor. The millennium development goals report 2015; 2015. http://www.un.org/millenniumgoals/2015_MDG_Report/pdf/ MDG\%202015\%20rev\%20\%28July\%201\%29.pdf. Accessed 3 Apr 2017.

8. The World Bank. Mortality rate, neonatal (per 1,000 live births). 2016. http:// data.worldbank.org/indicator/SH.DYN.NMRT. Accessed 3 Apr 2017.

9. Nankabirwa V, Tumwine JK, Tyllskar T, Nankunda J, Sommerfelt H, for the PROMISE EBF Research Consortium. Perinatal mortality in eastern Uganda: a community based prospective cohort study. PLoS One. 2011;6(5):e19674. https://doi.org/10.1371/journal.pone.0019674.

10. Ministry of Gender, Labour and Social Development and UNICEF-UGANDA Situation analysis of child poverty and deprivation in Uganda. Child Poverty and Deprivation Report, 2014. https://www.unicef.org/esaro/2014-UNICEFUganda-Child-Poverty-and-Deprivation.pdf. Accessed 3 Apr 2017.

11. Pettit D. World Health Organisation. Clean care is safer care. 2016. http:// www.who.int/gpsc/pittet message/en/. Accessed 3 Apr 2017.

12. Mugalu J, Nakakeeto MK, Kiguli S, Kaddu-Mulindwa DH. Aetiology, risk factors and immediate outcome of bacteriologically confirmed neonatal septicaemia in Mulago Hospital, Uganda. Afr Health Sci. 2006;6(2):120-6.

13. Mathur P. Hand hygiene: back to the basics of infection control. Indian J Med Res. 2011 Nov;134(5):611-20. https://doi.org/10.4103/0971-5916.90985.

14. Ditai J, Abeso J, Mudoola M, Faragher B, Adengo M, Richards JD, et al. A pilot cluster randomised trial of alcohol-based hand rub to prevent community neonatal sepsis in rural Uganda. BJOG. 2016;123(s2):121-9.

15. World Health Organisation. Country Cooperation Strategy at a glance. Uganda; 2014. http://apps.who.int/iris/bitstream/10665/136975/1/ccsbrief_ uga_en.pdf. Accessed 3 Apr 2017

16. Hotchkiss DR, Diana ML, Forfeit KG. How can routine health information systems improve health systems functioning in low- and middle- income countries? Assessing the evidence base. Adv Health Care Manag. 2012;12: 25-58. https://doi.org/10.1108/S1474-8231(2012)0000012006.

17. Owa JA, Olusanyu O. Neonatal bacteraemia in Wesley Guild hospital, llesha, Nigeria. Ann Trop Paediatr. 1988;8(2):80-4.

18. Mondal GP, Raghavan M, Vishnu Bhat B, Srinivasan S. Neonatal septicaemia among inborn and outborn babies in a referral hospital. Indian J Pediatr. 1991;58:529. https://doi.org/10.1007/BF02750936.

19. Zaida AKM, Thaver D, Ali SA, Khan TA. Pathogens associated with sepsis in newborns and young infants in developing countries. Paediatr Infect Dis J. 2009;28(1):S10-8. https://doi.org/10.1097/INF.0b013e3181958769.

20. Waters D, Jawad I, Ahmad A, Lukšić I, Nair H, Zgaga L, et al. Aetiology of community-acquired neonatal sepsis in low- and middle-income countries. J Glob Health. 2011;1(2):154-70.

21. Plettig R, Nowak A, Balau V, Hahnenkamp K, Usichenko T. Prospective comparison of a PCR assay and a microbiological culture technique for identification of pathogens from blood and non-blood samples in septic patients. J Intensive Care. 2015;3:51. https://doi.org/10.1186/s40560-0150116-1.

22. Seale AC, Obiero CW, Berkley JA. Rational development of guidelines for management of neonatal sepsis in developing countries. Curr Opin Infect Dis. 2015;28(3):225-30. https://doi.org/10.1097/QC0.0000000000000163.

23. Ganatra HA, Zaidi AKM. Neonatal infections in the developing world. Semin Perinatol. 2010:34:6. https://doi.org/10.1053/j.semperi.2010.09.004.

24. The Young Infants Clinical Signs Study Group. Clinical signs that predict severe illness in children under 2 months: a multicentre study. Lancet. 2008; 371(9607):135-42. https://doi.org/10.1016/S0140-6736(08)60106-3.

25. World Health Organisation. Integrated management of childhood illnesses chart booklet. 2008. https://www.scribd.com/document/8585609/IMCIChart-Booklet-2008-edition-Integrated-Management-of-Childhood-IIInessWorld-Health-Organization-WHO-United-Nations-International-Children-sEme. Accessed 11 Nov 2018.

26. Baqui AH, Arifeen SE, Rosen HE, Mannan I, Rahman SM, Al-Mahmud AB, et al. Community-based validation of assessment of newborn illnesses by trained community health workers in Sylhet district of Bangladesh. Tropical Med Int Health. 2009;14(12):1448-56. https://doi.org/10.1111/j.1365-3156. $2009.02397 \times$ Epub 2009 Oct 5 .
27. Baqui AH, El-Arifeen S, Darmstadt GL, Ahmed S, Williams EK, Seraji HR, et al. Effect of community-based newborn-care intervention package implemented through two service-delivery strategies in Sylhet district, Bangladesh: a cluster-randomised controlled trial. Lancet. 2008;371:1936-44. https://doi.org/10.1038/jp.2015.139.

28. World Health Organisation: The partnership for maternal, newborn and child health. Newborn Death and IIIness. 2011. http://www.who.int/pmnch/ media/press_materials/fs/fs_newborndealth_illness/en/. Accessed 3 Apr 2017

29. World Health Organisation: Handbook $\mathrm{IMCl}$ integrated management of childhood illness. 2005. http://apps.who.int/iris/bitstream/10665/42939/1/ 9241546441.pdf. Accessed 3 Apr 2017.

30. Kananura RM, Tetui M, Mutebi A, Bua JN, Waiswa P, Kiwanuka SN, et al. The neonatal mortality and its determinants in rural communities of Eastern Uganda. Reprod Health. 2016:13:13. https://doi.org/10.1186/s12978-0160119-y Accessed 3 Apr 2017

31. Kozuki N, Guenther T, Vaz L, Moran A, Soofi SB, Kayemba CN, et al. A systematic review of community-to-facility neonatal referral completion rates in Africa and Asia. BMC Public Health. 2015;15(989). https://doi.org/10. 1186/s12889-015-2330-0.

32. Lee AC, Chandran A, Herbert HK, Kozuki N, Markell P, Shah R, et al. Treatment of infections in young infants in low- and middle-income countries: a systematic review and meta-analysis of frontline health worker diagnosis and antibiotic access. PLoS Med. 2014;11(10):e1001741. https://doi. org/10.1371/journal.pmed.1001741.

33. Kibuule D, Kagoya HR, Godman B. Antibiotic use in acute respiratory infections in under-fives in Uganda: findings and implications. Expert Rev Anti-Infect Ther. 2016;14(9):863-72. https://doi.org/10.1080/14787210.2016. 1206468.

34. Huynh BT, Padget M, Garin B, Herindrainy P, Kermorvant-Duchemin E, Watier $\mathrm{L}$, et al. Burden of bacterial resistance among neonatal infections in low income countries: how convincing is the epidemiological evidence? BMC Infect Dis. 2015:15:127. https://doi.org/10.1186/s12879-015-0843-X.

35. Lubell Y, Ashley EA, Turner C, Turner P, White NJ. Susceptibility of community-acquired pathogens to antibiotics in Africa and Asia in neonates - an alarmingly short review. Tropical Med Int Health. 2011;16(2): 145-51. https://doi.org/10.1111/j.1365-3156.2010.02686.x
Ready to submit your research? Choose BMC and benefit from:

- fast, convenient online submission

- thorough peer review by experienced researchers in your field

- rapid publication on acceptance

- support for research data, including large and complex data types

- gold Open Access which fosters wider collaboration and increased citations

- maximum visibility for your research: over $100 \mathrm{M}$ website views per year

At $\mathrm{BMC}$, research is always in progress.

Learn more biomedcentral.com/submission 